\title{
Avaliação do desenvolvimento motor de recém-nascidos prematuros extremos e moderados em uma unidade de terapia intensiva neonatal
}

\section{Evaluation of engine development of extreme and moderate premature newborns in a neonatal intensive therapy unit}

\author{
Kelen Salvagni' ${ }^{1}$ Laís Rodrigues Gerzson ${ }^{2}$, Carla Skilhan de Almeida ${ }^{3}$ \\ http://dx.doi.org/10.11606/issn.2238-6149.v30i2p77-85
}

\begin{abstract}
Salvagni K, Gerzson LR,Almeida CS. Avaliação do desenvolvimento motor de recém-nascidos prematuros extremos e moderados. Rev Ter Ocup Univ São Paulo. 2020 maio-ago.;30(2):77-85.
\end{abstract}

RESUMO: Objetivo: avaliar o desenvolvimento motor de recémnascidos prematuros extremos e comparar ao desenvolvimento motor dos recém-nascidos prematuros moderados, em uma UTI Neonatal. Métodos: estudo transversal onde foram avaliados 16 neonatos, cinco prematuros extremos e 11 prematuros moderados, classificados pelo Test of Infant Motor Performance. Resultados: Não houve diferença significativa no desempenho motor entre o grupo de prematuros extremos e moderados, entretanto, a prematuridade extrema reduziu 4,74 pontos no escore. Na correlação entre os fatores de risco e o desenvolvimento motor, o uso de ventilação mecânica não invasiva e o Apgar no $1^{\mathrm{o}}$ minuto foram associados estatisticamente com o desempenho motor atípico. As demais variáveis não apresentaram correlação significativa com o desenvolvimento motor. Conclusão: não houve associação entre a idade gestacional e o desenvolvimento motor de neonatos prematuros.

DESCRITORES: Recém-nascido prematuro; Desenvolvimento infantil; Unidades de terapia intensiva neonatal.
Salvagni K, Gerzson LR, Almeida CS. Evaluation of engine development of extreme and moderate premature newborns. Rev Ter Ocup Univ São Paulo. 2020 May-Aug.;30(2):77-85.

ABSTRACT: Objective: to evaluate the motor development of preterm infants and to compare motor development of the preterm infants in a neonatal intensive care unit. Methods: a cross-sectional study in which 16 neonates were evaluated, five of which were premature and 11 were premature, classified by the test of infant motor performance. Results: there was no significant difference in motor performance between the group of extreme and moderate preterm infants, however, extreme prematurity reduced 4.74 points in the score. In the correlation between risk factors and motor development, the use of non-invasive mechanical ventilation and apgar at 1 minute were statistically associated with atypical motor performance. The other variables did not present a significant correlation with motor development. Conclusion: there was no association between gestational age and the motor development of preterm infants.

KEYWORDS: Infant, premature; Child development; Intensive care units, neonatal.

1. Fisioterapeuta, Hospital Materno Infantil Presidente Vargas, Porto Alegre, RS, Brasil. https://orcid.org/0000-0002-5457-9156. Email: kelensalvagni@gmail.com.

2. Programa de Pós-Graduação Saúde da Criança e do Adolescente, Universidade Federal do Rio Grande do Sul, Porto Alegre, RS, Brasil. https://orcid.org/0000-0002-0911-9820. Email: gerzson.lais@yahoo.com.br.

3. Departamento de Fisioterapia, Educação Física e Dança, Universidade Federal do Rio Grande do Sul, Porto Alegre, RS, Brasil. https://orcid.org/0000-0003-1271-2876. Email: carlaskilhan@gmail.com.

Endereço para correspondência: Carla Skilhan de Almeida. Rua Felizardo, 750 - Jardim Botânico. Porto Alegre, RS. CEP: $90690-200$. E-mail: carlaskilhan@gmail.com. 


\section{INTRODUÇÃO}

$\mathrm{O}_{\mathrm{t}}^{\mathrm{s}}$ desenvolvimento motor é caracterizado pela transformação progressiva no desempenho funcional do indivíduo durante sua vida, decorrente da interação entre os fatores biológicos, as necessidades das atividades e as influências ambientais. É um processo constante, que tem origem na concepção do ser e se encerra apenas com o óbito ${ }^{1}$. A princípio, consideravase que as alterações no desempenho motor dos sujeitos eram determinadas unicamente pela maturação do sistema nervoso central. Atualmente, entende-se que a evolução no comportamento motor é dinâmica, e pode ser influenciada por estímulos externos (ambiente e a tarefa) ${ }^{2}$.

Existem vários fatores considerados indicadores de risco para o desenvolvimento infantil, classificados como: risco biológico (baixo peso ao nascer, prematuridade, ventilação mecânica, hemorragia peri-intraventricular, exposição pré-natal de álcool e drogas); risco estabelecido (hidrocefalia, microcefalia, anomalias cromossômicas e musculoesqueléticas); e risco ambiental/social (vulnerabilidade social, anomalias no estado comportamental) ${ }^{3}$. Os prejuízos para o desenvolvimento são potencializados quando há associação dessas condições de risco ${ }^{4}$.

Um dos fatores de risco preponderante na UTI Neonatal é a prematuridade, determinada pelo nascimento do feto antes de completar sua maturidade cronológica. Os recém-nascidos são considerados prematuros quando o nascimento ocorre antes da gestação completar 37 semanas e são classificados conforme seu nível de maturidade. Os prematuros limítrofes são aqueles nascidos entre 35 a 37 semanas de gestação; os prematuros moderados, entre 30 e 35 semanas; e prematuros extremos são aqueles nascidos com 30 semanas ou menos ${ }^{5}$.

Dados da pesquisa Nascer no Brasil constataram que a taxa de prematuridade no país é de $11,5 \%$, o que coloca o país em $10^{\circ}$ lugar na lista internacional de nascimentos prematuros $^{6}$. No município de Porto Alegre, o índice de prematuridade é de $11,34 \%$ do total de nascimentos ${ }^{7}$.

Nas últimas décadas, o aperfeiçoamento científico e tecnológico da assistência aos neonatos graves fez com que o índice de sobrevida dessa população aumentasse significativamente ${ }^{8}$. Muitos desses bebês necessitam de cuidados especiais, sendo que a internação em Unidade de Terapia Intensiva (UTI) Neonatal torna-se indispensável para sua sobrevivência. No entanto, a permanência nesse ambiente, onde ficam expostos à estimulação hostil excessiva (procedimentos dolorosos, ruídos e excesso de luminosidade) pode provocar alterações no desenvolvimento motor, devido a sua imaturidade neurofisiológica?.
A detecção precoce de atrasos no desenvolvimento possibilita intervenções efetivas, prevenindo distúrbios do desenvolvimento e promovendo o adequado desempenho motor dos lactentes ${ }^{10}$, ou seja, é uma fase onde a plasticidade neural está acelerada e há aquisição de inúmeras habilidades motoras. O diagnóstico precoce de alterações do desenvolvimento é imprescindível para que a intervenção possa iniciar o mais precocemente possível, otimizando os resultados interventivos, melhorando as competências funcionais e diminuindo os prejuízos na vida futura destes bebês ${ }^{11,12}$.

Acredita-se que essa deteç̧ão precoce pode acontecer já em uma UTI Neonatal. Nosso estudo foi realizado em uma UTI Neonatal de um hospital público de referência de Porto Alegre, no qual realiza boas práticas em seu campo de ação. O estudo foi proposto pela fisioterapia do serviço de Residência Integrada Multiprofissional em Saúde da CriançaViolência e Vulnerabilidades A dúvida que motivou o grupo a realizar o estudo seria se os prematuros extremos teriam uma alteração no desenvolvimento e, em caso positivo, se essa alteração seria maior que de recém-nascidos prematuros moderados. Diante do exposto, o objetivo deste estudo foi avaliar o desenvolvimento motor de recém-nascidos prematuros extremos e comparar ao desenvolvimento motor de recém-nascidos prematuros moderados, em uma UTI Neonatal de referência de Porto Alegre, RS.

\section{PROCEDIMENTOS METODÓLOGICOS}

\section{Delineamento e Amostra}

Trata-se de um estudo transversal, onde foram avaliados recém-nascidos prematuros extremos e moderados, internados na UTI Neonatal do Hospital Materno Infantil Presidente Vargas entre os meses de agosto a outubro de 2018.

Foram incluídos nesta pesquisa neonatos prematuros nascidos até 35 semanas de idade gestacional, com idade corrigida mínima de 34 semanas no momento da avaliação, que estivessem hemodinamicamente estáveis e respirando espontaneamente, mediante autorização dos pais ou responsáveis, através da assinatura do Termo de Consentimento Livre e Esclarecido. Foram excluídos do estudo os bebês que (a) apresentaram malformações congênitas graves, (b) distúrbios cromossômicos, (c) os que estavam em ventilação mecânica e (d) os que não tiveram o consentimento dos pais ou responsáveis.

O estudo foi submetido e aprovado pelo Comitê de Ética em Pesquisa do Hospital Materno Infantil Presidente Vargas (CEP-HMIPV), com número CAEE 92490218.6.0000.5329. 


\section{Instrumentos}

Os dados dos pacientes avaliados e informações clínicas foram coletados em prontuário físico e registrados em instrumento de coleta de dados criado pelas pesquisadoras. Esse prontuário fica disponível nas Unidades de Tratamento Intensivo do Hospital enquanto o bebê estiver nesta unidade. Após alta hospitalar, o prontuário se localiza no setor de arquivos.

Os bebês foram avaliados com o Test of Infant Motor Performance (TIMP), que avalia o controle da postura e a movimentação espontânea e seletiva do recém-nascido, fundamentais para o desenvolvimento motor funcional. O TIMP engloba 42 itens de avaliação agrupados em duas seções, sendo 13 itens observados, que analisam o movimento espontâneo do bebê e 29 itens eliciados, que avaliam as respostas motoras nas posições supino, prono, sentado e em pé. Cada item observado recebe escore um e quando o item não for observado recebe escore zero. Os itens eliciados são pontuados de zero a seis, conforme a resposta do bebê aos movimentos desencadeados pelo avaliador. O escore total do teste é a soma da pontuação dos itens observados com os itens eliciados, o qual será comparado à tabela de padrões de desempenho motor onde os bebês são classificados em um dos quatro níveis: média, média baixa, abaixo da média e muito abaixo da média ${ }^{13,14}$.

Os participantes foram avaliados no instante em que apresentavam os estágios quatro (acordado) ou cinco (totalmente acordado) da Escala de Avaliação do Estado de Sono e Vigília adaptada de Brazelton. Os materiais utilizados para a avaliação foram: um chocalho, uma bola vermelha, um brinquedo sonoro e um pano macio. Os recém-nascidos foram submetidos a uma única avaliação, por fisioterapeuta treinada, com duração média de 30 minutos, no momento em que atingiram os critérios de inclusão.

As mães dos recém-nascidos que foram avaliados na presente pesquisa receberam orientações sobre estimulação motora, posicionamento e atividades a serem realizadas no domicílio com seus bebês, incluindo um folder com informações e ilustrações. Os neonatos considerados atípicos foram encaminhados para intervenção motora no momento da alta.

Todos os participantes receberam alta com acompanhamento agendado para o ambulatório de pediatria do hospital e foi reforçada para os responsáveis a importância de dar sequência ao acompanhamento e às avaliações para garantirmos a detecção precoce caso haja alguma suspeita de atraso no desenvolvimento motor.

\section{Análise dos Dados}

Para a análise estatística, as variáveis quantitativas (idade gestacional, peso ao nascer, APGAR no $1^{\circ} \mathrm{e}$ $5^{\circ}$ minuto, idade materna, peso atual, dias de ventilação mecânica, dias ventilação mecânica não-invasiva, tempo de internação e escore TIMP) foram descritas por média e desvio padrão ou mediana e amplitude de variação. As variáveis categóricas (peso ao nascer, sexo, tipo de parto, uso de ventilação mecânica, uso de ventilação mecânica não invasiva, ocorrência de convulsões, suporte ventilatório durante avaliação, classificação e avaliação TIMP) foram descritas por frequências absolutas e relativas. Para comparar médias, o teste t-student foi aplicado. Em caso de assimetria, o teste de Mann-Whitney foi utilizado. Na comparação de proporções, o teste exato de Fisher foi aplicado. Para controle de fatores confundidores, o modelo de Regressão Linear foi aplicado. O critério para a entrada da variável no modelo multivariado foi de que a mesma apresentasse um valor $\mathrm{p}<0,20$ na análise bivariada. Foi apresentado o coeficiente de regressão angular que mede o efeito (b) em conjunto com o intervalo de $95 \%$ de confiança. Também foi calculada a Razão de Prevalências (RP) em conjunto com o intervalo de $95 \%$ de confiança. O nível de significância adotado foi de $5 \%(\mathrm{p}<0,05)$ e as análises foram realizadas no programa Statistical Package for the Social Sciences (SPSS) versão 21.0.

\section{RESULTADOS}

\section{Composição da amostra}

Durante os meses de agosto a outubro internaram na UTI Neonatal 128 recém-nascidos; destes 66 nasceram a termo, 61 foram prematuros e um teve nascimento pós termo. Dos que nasceram prematuros 13 foram prematuros extremos, 24 prematuros moderados e 24 prematuros limítrofes.

Dos 13 prematuros extremos, dois foram a óbito, dois os pais não consentiram com a avaliação, dois não conseguimos contato com os responsáveis, um nasceu com malformações e um não atingiu os critérios de inclusão, totalizando oito excluídos da pesquisa, sendo avaliados cinco recém-nascidos prematuros extremos.

Dos 24 prematuros moderados, três nasceram com mal formações, dois permaneceram em isolamento de contato até o momento da alta, não podendo sair da 
incubadora para a realização da avaliação, um ficou em fototerapia até o momento da alta, também não sendo possível sair da incubadora, impossibilitando a participação na pesquisa, um não conseguimos contato com responsável e seis não atingiram os critérios de inclusão, totalizando 13 bebês excluídos da pesquisa, sendo avaliados 11 recém-nascidos prematuros moderados.

Dessa forma, a amostra foi composta por 16 recémnascidos, sendo cinco prematuros extremos (nascidos com 30 semanas ou menos) e 11 prematuros moderados (nascidos entre 30 e 35 semanas).

\section{Perfil da amostra}

Com relação ao perfil geral da amostra, cinco dos bebês avaliados eram prematuros extremos $(31,3 \%)$ e 11 prematuros moderados (68,8\%), 14 eram do sexo masculino $(87,5 \%)$ e dois do sexo feminino (12,5\%), cinco necessitaram de ventilação mecânica invasiva $(31,3 \%), 11$ fizeram uso de ventilação mecânica não invasiva (68,8\%), dez nasceram de parto cesáreo $(62,5 \%)$ e seis nasceram de parto vaginal $(37,5 \%)$.

A Tabela 1 apresenta a caracterização da amostra dividida por grupos.

Tabela 1 - Caracterização da amostra

\begin{tabular}{|c|c|c|c|}
\hline Variáveis & $\begin{array}{c}\text { Prematuridade Extrema } \\
(n=5)\end{array}$ & $\begin{array}{c}\text { Prematuridade Moderada } \\
(\mathrm{n}=11)\end{array}$ & $\mathbf{p}$ \\
\hline Idade Gestacional $(\mathrm{sem})-$ média $\pm \mathrm{DP}$ & $28,6 \pm 1,5$ & $33,5 \pm 1,2$ & $<0,001$ \\
\hline Peso ao nascer $(\mathrm{g})-$ média $\pm \mathrm{DP}$ & $1166 \pm 301$ & $1917 \pm 353$ & 0,001 \\
\hline Classificação do peso ao nascer - n (\%) & & & 0,002 \\
\hline Extremo baixo peso & $2(40,0)$ & $0(0,0)$ & \\
\hline Muito baixo peso & $3(60,0)$ & $1(9,1)$ & \\
\hline Baixo peso & $0(0,0)$ & $10(90,9)$ & \\
\hline Sexo-n $(\%)$ & & & 1,000 \\
\hline Masculino & $4(80,0)$ & $10(90,9)$ & \\
\hline Feminino & $1(20,0)$ & $1(9,1)$ & \\
\hline APGAR $1^{\circ}$ minuto - média (min-max) & $5(2-8)$ & $8(3-9)$ & 0,027 \\
\hline APGAR $5^{\circ}$ minuto - média (min-max) & $8(7-9)$ & $9(7-10)$ & 0,069 \\
\hline Idade materna (anos) - média \pm DP & $29,6 \pm 7,2$ & $30,3 \pm 7,2$ & 0,865 \\
\hline Parto - n (\%) & & & 1,000 \\
\hline Cesáreo & $3(60,0)$ & $7(63,6)$ & \\
\hline Vaginal & $2(40,0)$ & $4(36,4)$ & \\
\hline
\end{tabular}

sem: semanas; g: gramas; DP: desvio padrão; min: mínimo; máx: máximo; n: número

\section{Correlações}

Para correlacionar as variáveis foi realizado modelo multivariado para o escore bruto, com controle de fatores confundidores, através do modelo de Regressão Linear, entrando no modelo: prematuridade, peso atual, Apgar $1^{\circ}$ e $5^{\circ}$ minutos, uso de ventilação mecânica invasiva e ventilação mecânica não invasiva, dias de internação e ventilação. Após o ajuste, a prematuridade extrema não ficou associada estatisticamente com o escore de desenvolvimento motor $(b=-4,74$; IC $95 \%$ : $-18,1$ a 8,$6 ; \mathrm{p}=0,451)$, apesar da prematuridade extrema reduzir, em média, 4,74 pontos no escore.

Os desfechos das variáveis analisadas, conforme o grau de prematuridade, estão apresentados na Tabela 2. 
Salvagni K, et al. Avaliação do desenvolvimento motor de recém-nascidos. Rev Ter Ocup Univ São Paulo. 2020 maio/ago.;30(2):77-85.

Tabela 2 - Desfechos conforme grau de prematuridade.

\begin{tabular}{|c|c|c|c|}
\hline Variáveis & $\begin{array}{l}\text { Prematuridade } \\
\text { Extrema }(n=5)\end{array}$ & $\begin{array}{c}\text { Prematuridade } \\
\text { Moderada }(n=11)\end{array}$ & $\mathbf{p}$ \\
\hline Peso atual $(\mathrm{g})-$ média $\pm \mathrm{DP}$ & $2927 \pm 728$ & $2105 \pm 195$ & 0,064 \\
\hline Uso de VM - n (\%) & $4(80,0)$ & $1(9,1)$ & 0,013 \\
\hline Dias de VM - mediana (min-max) & $26,5(2-57)$ & $36(36-36)$ & 1,000 \\
\hline Uso de VMNI - n (\%) & $5(100)$ & $6(54,5)$ & 0,119 \\
\hline Dias de VMNI - mediana (min-max) & $6(1-12)$ & $2,5(1-5)$ & 0,126 \\
\hline Convulsão - n (\%) & $0(0,0)$ & $1(9,1)$ & 1,000 \\
\hline Tempo de internação (dias) - mediana (min-max) & $75(55-165)$ & $25(9-66)$ & 0,002 \\
\hline Ventilação durante avaliação - n (\%) & & & 0,083 \\
\hline Ar ambiente & $3(60,0)$ & $11(100)$ & \\
\hline Oxigênio por cateter nasal & $2(40,0)$ & $0(0,0)$ & \\
\hline Escore TIMP - média \pm DP & $47,4 \pm 12,2$ & $39,6 \pm 6,2$ & 0,108 \\
\hline Classificação - n (\%) & & & 0,330 \\
\hline Média & $0(0,0)$ & $2(18,2)$ & \\
\hline Média baixa & $2(40,0)$ & $3(27,3)$ & \\
\hline Abaixo da média & $2(40,0)$ & $6(54,5)$ & \\
\hline Muito abaixo da média & $1(20,0)$ & $0(0,0)$ & \\
\hline Avaliação - n (\%) & & & 0,575 \\
\hline Típico & $0(0,0)$ & $2(18,2)$ & \\
\hline Suspeito & $2(40,0)$ & $3(27,3)$ & \\
\hline Atípico & $3(60,0)$ & $6(54,5)$ & \\
\hline
\end{tabular}

g: gramas; DP: desvio padrão; VM: ventilação mecânica, VMNI: ventilação mecânica não invasiva; min: mínimo; máx: máximo; n: número.

Foi efetuado modelo multivariado para determinar os preditores de desenvolvimento atípico, com controle de fatores confundidores, através do modelo de Regressão de Poisson, entrando no modelo: prematuridade, peso atual, Apgar $1^{\circ}$ e $5^{\circ}$ minuto, uso de ventilação mecânica invasiva e ventilação mecânica não invasiva, dias de internação e ventilação. Após o ajuste, a prematuridade extrema não ficou associada estatisticamente com o escore de desenvolvimento motor ( $\mathrm{RP}=0,93$; IC 95\%: 0,39-2,19; $\mathrm{p}=0,865)$. Os fatores que permaneceram associados estatisticamente com o desenvolvimento motor atípico foram: uso de VMNI $(\mathrm{RP}=5,05$; IC 95\%: 1,01 a 25,1; $\mathrm{p}=0,048)$, sendo que o mesmo aumenta a prevalência de desenvolvimento atípico em 5,05 vezes, como pode ser visualizado na Figura 1.

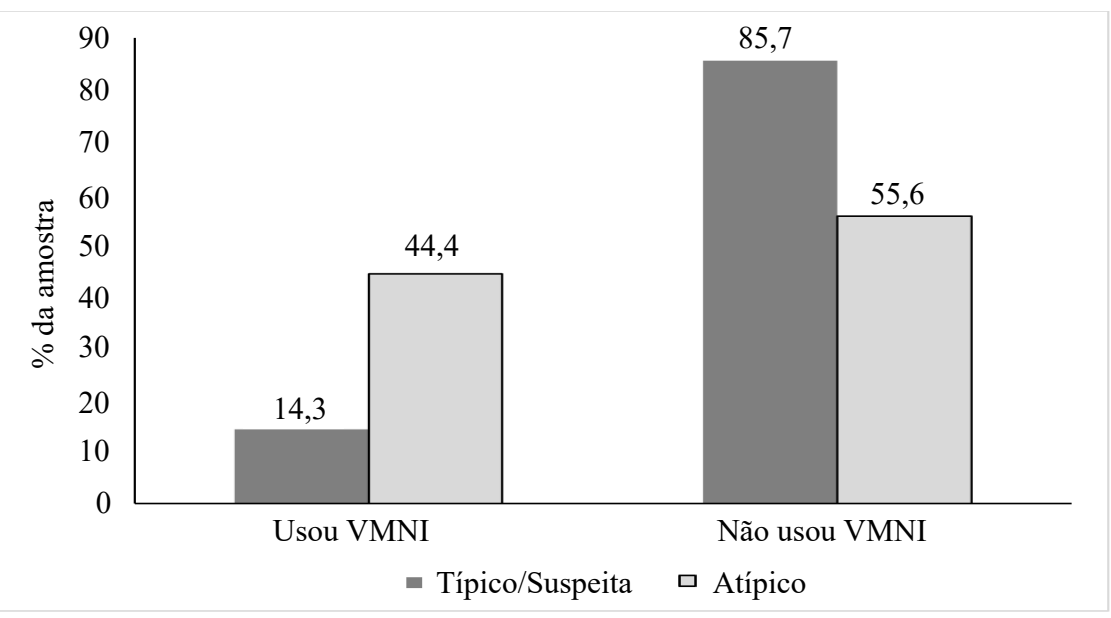

Figura 1 - Desenvolvimento motor conforme uso ou não de ventilação mecânica não invasiva 
Também o Apgar no $1^{\circ}$ minuto permaneceu associado $(\mathrm{RP}=0,71 ; \mathrm{IC} 95 \%$ : 0,55 a 0,91; $\mathrm{p}=0,007)$, sendo que um ponto a mais no Apgar do $1^{\circ}$ minuto, há uma redução média de $29 \%$ na probabilidade de desenvolvimento atípico, como pode ser visualizado na Figura 2.

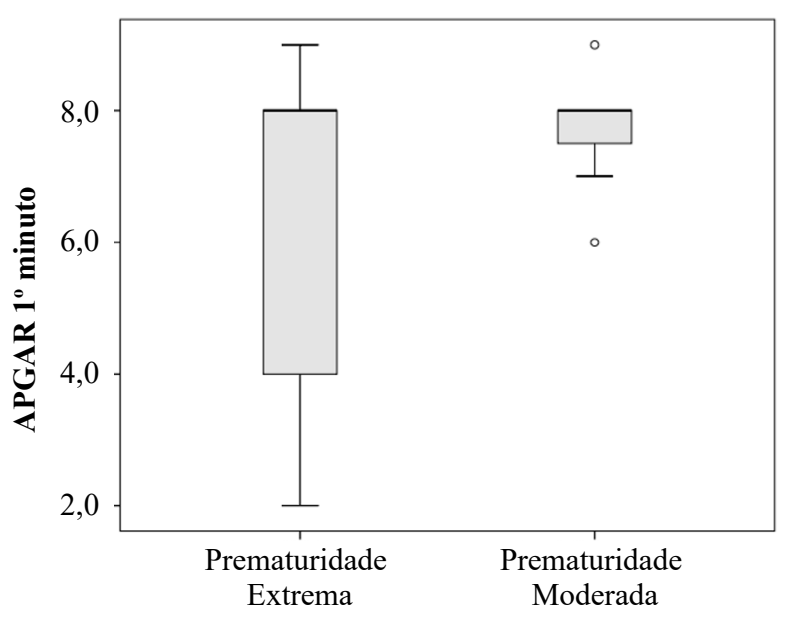

Grupo

Figura 2 - Apgar no $1^{\circ}$ minuto conforme tipo de prematuridade

No grupo de prematuros extremos, dois bebês (40\%) foram classificados na média baixa, dois $(40 \%)$ foram classificados abaixo da média e um bebê $(20 \%)$ teve o pior desempenho e foi classificado como muito abaixo da média. Nenhum dos participantes deste grupo atingiu a classificação na média de acordo com o desempenho motor padrão para a idade corrigida. Sendo assim, dois bebês ( $40 \%$ ) foram considerados suspeitos para atraso no desenvolvimento motor e os outros três $(60 \%)$ ) foram classificados como atípicos.

No grupo de prematuros moderados, dois bebês $(18,2 \%)$ receberam a melhor classificação da TIMP, sendo considerados na média para a idade corrigida, ou seja, desenvolvimento motor típico; três bebês $(27,3 \%)$ foram classificados na média baixa, avaliados como suspeitos, e seis bebês $(54,5 \%)$ receberam classificação abaixo da média, de acordo com a tabela de escores da TIMP, sendo o desempenho motor considerado atípico. Nenhum recém-nascido deste grupo ficou classificado como muito abaixo da média.

\section{DISCUSSÃO}

O presente estudo comparou a classificação do desenvolvimento motor do grupo de recém-nascidos prematuros extremos com o grupo de prematuros moderados não encontrando diferença significativa no desempenho dos grupos. Apesar disso, é importante ressaltar que a prematuridade extrema reduziu, em média, 4,74 pontos no escore. Esse resultado corrobora com a pesquisa de Santos ${ }^{15}$, a qual demonstrou que apesar de não ter encontrado associação significativa entre a idade gestacional e o desenvolvimento motor, existe a observação de que quanto maior for o grau de prematuridade, maior pode ser o comprometimento nas habilidades motoras.

Contrariamente a esse desfecho, um estudo realizado no Japão demonstrou que $50 \%$ dos prematuros extremos nascidos entre 22 e 23 semanas de idade gestacional e 30\% dos que nasceram entre 24 e 25 semanas apresentam déficits no neurodesenvolvimento, quando avaliados aos três anos de idade cronológica, demonstrando que os atrasos no desenvolvimento estão relacionados à idade gestaciona ${ }^{16}$.

Apesar de não ter havido diferença significativa na classificação do desempenho motor entre o grupo de prematuros extremos e o grupo de prematuros moderados, é indispensável destacar que a maioria dos recém-nascidos avaliados - três prematuros extremos $(60 \%)$ e seis moderados (54,5\%) - ficaram abaixo da média esperada para a idade corrigida, sinalizando que estes bebês representam um grupo de risco para atraso no desenvolvimento motor. Estudos revelam que metade dos recém-nascidos extremamente prematuros apresenta déficits importantes no desenvolvimento neurológico a curto e longo prazo ${ }^{17}$.

$\mathrm{Na}$ correlação entre os fatores de risco biológicos e o desenvolvimento motor, observou que o uso de ventilação mecânica não invasiva foi associado estatisticamente com o desempenho motor atípico, sendo que a necessidade de suporte ventilatório aumentou a prevalência de desenvolvimento atípico em 5,05 vezes. Resultados semelhantes foram verificados no estudo de Nicolau et al. ${ }^{16}$ no qual foram avaliados 69 recém-nascidos prematuros de alto risco, e verificou-se que o uso de ventilação mecânica, tanto invasiva quanto não invasiva teve influência no desenvolvimento motor dos bebês, sendo que os que necessitaram de suporte ventilatório por mais tempo obtiveram classificação abaixo da média e muito abaixo da média no TIMP.

Araújo et al. ${ }^{18}$ também evidenciaram que o uso de ventilação mecânica apresentou associação significativa com o desenvolvimento motor de bebês prematuros internados em uma UTI neonatal.

Uma possível explicação para esta associação é que a produção de radicais livres após o nascimento é intensificada pelo uso de oxigênio terapêutico. De acordo com o estudo de French et al. ${ }^{19}$, o estresse oxidativo pode ser um coadjuvante para a hipomielinização difusa observada na lesão da matriz branca periventricular, que está relacionada à lesão neurológica crônica em neonatos prematuros, o que pode causar atraso no desenvolvimento motor. 
Além disso, observa-se uma limitação na variação de posições e restrição da movimentação espontânea dos bebês submetido à ventilação mecânica ${ }^{18}$. Para facilitar o manuseio da equipe e otimizar os cuidados necessários à sobrevivência do neonato, muitas vezes o posicionamento utilizado não é o que mais beneficia o desenvolvimento motor.

Outra explicação possível para os achados é o fato de que as crianças que utilizam suporte ventilatório frequentemente apresentam alterações hemodinâmicas e diminuição da oxigenação tecidual, que podem contribuir para lesões neurológicas ${ }^{20}$.

Outra variável que teve associação com a performance motora foi o Apgar no $1^{\circ}$ minuto, cada ponto a mais no Apgar representou uma redução média de 29\% na probabilidade de desenvolvimento atípico. Um estudo de revisão sistemática sobre o desenvolvimento motor de prematuros evidenciou que em algumas pesquisas o escore de Apgar esteve associado a um desfecho motor pobre $^{21}$. Distintivamente, Santos ${ }^{15}$ não encontrou associação significativa entre o índice de Apgar e o desenvolvimento motor de crianças prematuras em idade pré-escolar.

O índice de Apgar avalia o estado clínico dos recémnascidos imediatamente após o nascimento, bem como sua adaptação ao ambiente extrauterino. Segundo estudos, este índice isoladamente não é preditor do estado neurológico do neonato, mas é uma previsão da morbidade e mortalidade do bebêê22.

As demais variáveis analisadas não apresentaram correlação significativa com o desenvolvimento motor dos recém-nascidos avaliados. Diversos estudos já demonstraram que há uma preponderância dos fatores ambientais sobre os biológicos, no que se refere ao desenvolvimento $\operatorname{motor}^{23}$. Essa evidência reforça a necessidade de encaminhamento precoce dos bebês com suspeita de atraso no desempenho motor para programas de intervenção, de forma que se possa agir previamente às alterações funcionais, melhorando a perspectiva do desenvolvimento e a qualidade de vida dessas crianças, possibilitando a esses bebês mais oportunidade de alcançar um desenvolvimento funcional adequado.

Uma meta-análise que incluiu estudos publicados desde 1980 até 2015 demonstrou que os efeitos das intervenções motoras diminuem ao longo do tempo, revelando a importância da detecção precoce, considerando que os efeitos mais influentes foram observados quando a avaliação do desenvolvimento motor e intervenção iniciaram antes dos seis meses de vida do lactente ${ }^{24}$.
A alta plasticidade apresentada pelos recém-nascidos durante o período em que se encontram internados na UTI Neonatal torna-os vulneráveis tanto aos estímulos nocivos como à estimulação benéfica. Desta forma, a intervenção motora precoce proporciona estímulos que favorecem o desenvolvimento motor adequado e atenuam os efeitos desfavoráveis do ambiente da UTI Neonata ${ }^{25}$.

O presente estudo apresentou como limitações o (a) tamanho amostral; e (b) ausência de acompanhamento longitudinal.

\section{CONCLUSÃO}

Observa-se que vários fatores peri e pós-natais podem interferir nos resultados do desempenho motor, por exemplo as complicações clínicas de cada recém-nascido, que podem trazer repercussões no desenvolvimento, associando fatores de risco e intensificando a probabilidade de haver atrasos nas aquisições motoras. Sendo assim, são necessários novos estudos com maior tempo de acompanhamento e agrupando os bebês de acordo com a gravidade e com os fatores de risco, para determinar quais são os mais impactantes no desenvolvimento motor.

Com base nos achados do presente estudo, concluiuse que existem muitas evidências que tornam os bebês prematuros crianças de risco para desenvolvimento motor atípico, principalmente quando há associação dos fatores de risco. Os resultados do estudo não apresentaram associação entre a idade gestacional e o desenvolvimento motor de neonatos prematuros, contudo, não devemos subestimar a necessidade de atenção para este fator de risco, principalmente pelo fato de que a prematuridade geralmente está associada a outras condições de risco que tornam o recém-nascido vulnerável a déficits no desenvolvimento motor. Sendo assim, é imprescindível que haja uma avaliação criteriosa o mais precocemente possível, proporcionando a possibilidade de intervir no desenvolvimento desses bebês, a fim de que possam ser estimulados para o melhor desempenho motor e funcional possível.

É de suma importância a necessidade de atenção para este fator de risco, especialmente porque a prematuridade geralmente está associada a outras condições de risco que tornam o recém-nascido vulnerável a déficits no desenvolvimento motor. Quanto antes for detectado alguma alteração no desenvolvimento, melhor pode basear as ações das equipes profissionais dedicadas à estimulação e ao acompanhamento do desenvolvimento infantil. Portanto, que este estudo pode basear futuros estudos nesta linha de abordagem.

Contribuições dos autores: $K S$ - Autor do estudo; $L R G, C S A$ - Autor e revisor do estudo. 


\section{REFERÊNCIAS}

1. Gallahue DL, Ozmun JC, Goodway J. Compreendendo o desenvolvimento motor: bebês, crianças, adolescentes e adultos. Porto Alegre: Artmed; 2013.

2. Pereira KRG, Saccani R, Valentini NC. Cognição e ambiente são preditores do desenvolvimento motor de bebês ao longo do tempo. Fisioter Pesqui. 2016;23(1):59-67. http://dx.doi.org/10.1590/1809-2950/14685223012016.

3. Perlroth NH, Branco CWC. Current knowledge of environmental exposure in children during the sensitive developmental periods. J Pediatr. 2017; 93(1):17-27. doi: 10.1016/j.jped.2016.07.002.

4. Borba LS, Saccani R, Valentini NC. Desenvolvimento motor de crianças nascidas pré-termo e a termo avaliadas com a escala motora infantil de Alberta. Temas Desenvolv. 2013;19(105):130-5.

5. Lotto CR, Linhares MBM. "Skin-to-skin" contact in pain prevention in preterm infants: systematic review of literature. Trends Psychol. 2018;26(4):1699-713. http://dx.doi.org/10.9788/tp2018.4-01pt.

6. Leonel F. Pesquisa Nascer no Brasil revela novos dados sobre prematuridade [citado 05 jul. 2018]. Disponível em: http://www.blog.saude.gov.br/index.php/promocao-dasaude/52044-pesquisa-nascer-no-brasil-revela-novos-dadossobre-prematuridade.

7. Panzenhagen H. Nascimentos prematuros têm dados alarmantes no Brasil [citado 05 jun. 2018]. Disponível em: $<$ http://www.camarapoa.rs.gov.br/noticias/nascimentosprematuros-tem-dados-alarmantes-no-brasil.

8. Victora CG, Aquino EM, Carmo Leal M, Monteiro CA, Barros FC, Szwarcwald CL. Maternal and child health in Brazil: progress and challenges. Lancet. 2011;377(9780):1863-76. doi: 10.1016/S0140-6736(11)60138-4.

9. Costa KSF, Beleza LDO, Souza LM, Ribeiro LM. Hammock position and nesting: comparison of physiological and behavioral effects in preterm infants. Rev Gaúcha Enferm. 2016;37(spe):e62554. doi: 10.1590/1983-1447.2016. esp. 62554 .

10. Danielli CR, Farias BL, Santos DAPB, Neves FE, Tonetta $\mathrm{MC}$, Gerzson LR, Almeida CS. Efeitos de um programa de intervenção motora precoce no desenvolvimento de bebês em um abrigo residencial. ConScientiae Saúde. 2016;15(3):370-7. https://doi.org/10.5585/conssaude.v15n3.6257.

11. Almeida CS, Paines AV, Almeida CB. Intervenção motora precoce ambulatorial para neonatos prematuros no controle postural. Rev Ciên Saúde. 2008;1(2):64-70. http://dx.doi.org/ 10.15448/1983-652X.2008.2.4200.

12. Byrne R, Noritz G, Maitre NL, NCH Early Developmental Group. Implementation of early diagnosis and intervention guidelines for cerebral palsy in a high-risk infant follow-up clinic. Pediatr Neurol. 2017;76:66-71. doi: 10.1016/j.pediatrneurol.2017.08.002.

13. Nicolau CM, Costa APBM, Hazime HO, Krebs VLJ. Desempenho motor em recém-nascidos pré-termo de alto risco. Rev Bras Crescimento Desenvolv Hum. 2011;21(2):327-34. Disponível em: http://pepsic.bvsalud.org/scielo.php?script=sci arttext\&pid=S0104-12822011000200015\&lng=pt\&tlng=pt.

14. Chiquetti EMS. Validação e normatização do "test of infant motor performance" (TIMP) para aplicação clínica e científica no Brasil [tese]. Porto Alegre: Universidade Federal do Rio Grande do Sul - UFRGS; 2018.

15. Santos PSP. Estudo do impacto da prematuridade, peso gestacional e outros fatores no desenvolvimento motor de crianças em idade pré-escolar [dissertação]. Lisboa: Universidade Técnica de Lisboa - UTL; 2012.

16. Ishii N, Kono Y, Yonemoto N, Kusuda S, Fujimura M, Neonatal Research Network Japan. Outcomes of infants born at 22 and 23 weeks' gestation. Pediatrics. 2013;132(1):62-71. doi: $10.1542 /$ peds.2012-2857

17. Jarjour IT. Neurodevelopmental outcome after extreme prematurity: a review of the literature. Pediatr Neurol. 2015;52(2):143-52. doi: 10.1016/j.pediatrneurol.2014.10.027

18. Araújo ATC, Eickmann SH, Coutinho SB. Fatores associados ao atraso do desenvolvimento motor de crianças prematuras internadas em unidade de neonatologia. Rev Bras Saúde Matern Infant. 2013;13(02):119-28. doi: 10.1016/j. pediatrneurol.2014.10.027.

19. French HM, Reid M, Mamontov P, Simmons RA, Grinspan JB. Oxidative stress disrupts oligodendrocyte maturation. J Neurosci Res. 2009;87(14):3076-87. doi: 10.1002/jnr.22139.

20. Limperopoulos C, Gauvreau KK, O'Leary H, Moore M, Bassan H, Eichenwald EC, et al. Cerebral hemodynamic changes during intensive care of preterm infants. Pediatrics. 2008;122(5):e1006-13. doi: 10.1542/peds.2008-0768.

21. Fuentefria RDN, Silveira RC, Procianoy RS. Motor development of preterm infants assessed by the Alberta Infant Motor Scale: systematic review article. J Pediatr. 2017;93(4):328-42. doi: 10.1016/j.jped.2017.03.003. 
Salvagni K, et al. Avaliação do desenvolvimento motor de recém-nascidos. Rev Ter Ocup Univ São Paulo. 2020 maio/ago.;30(2):77-85.

22. Oliveira TG, Freire PV, Moreira FT, Moraes JS, Arrelaro $\mathrm{RC}$, Rossi S, et al. Escore de Apgar e mortalidade neonatal em um hospital localizado na zona sul do município de São Paulo. Einstein. 2012;10(1):22-28. http://dx.doi.org/10.1590/ S1679-45082012000100006.

23. Borba LS, Pereira KRG, Valentini NC. Preditores do desenvolvimento motor e cognitivo de bebês de mães adolescentes e adultas. J Phys Educ. 2017;28(1):1-16. http://dx.doi.org/10.4025/jphyseduc.v28i1.2811.
24. Hughes AJ, Redsell SA, Glazebrook C. Motor development interventions for preterm infants: a systematic review and meta-analysis. Pediatrics. 2016;138(4):pii: e20160147. doi:10.1542/peds.2016-0147.

25. Guimarães CL, Reinaux CM, Botelho AC, Lima GM, Cabral Filho JE. Desenvolvimento motor avaliado pelo Test of Infant Motor Performance: comparação entre lactentes prétermo e a termo. Rev Bras Fisioter. 2011;15(5):357-62. http://dx.doi.org/10.1590/S1413-35552011005000021. 